\title{
Spin-Exchange Effects in Elastic Electron Scattering from Linear Triatomic Radicals
}

\author{
M. M. Fujimoto,${ }^{*, a}$ S. E. Michelin, ${ }^{b}$ F. Arretche, ${ }^{b}$ K.T. Mazon, ${ }^{b}$ M.-T. Lee ${ }^{c}$ and I. Iga ${ }^{c}$ \\ ${ }^{a}$ Departamento de Física, Universidade Federal do Paraná, 81531-990 Curitiba-PR, Brazil \\ ${ }^{b}$ Departamento de Física, Universidade Federal de Santa Catarina, \\ 88040-900 Florianópolis-SC, Brazil
}

${ }^{c}$ Departamento de Química, Universidade Federal de São Carlos, 13565-905 São Carlos- SP, Brazil

\begin{abstract}
Neste trabalho, apresentamos um estudo teórico sobre os efeitos de troca de spin no espalhamento elástico de elétrons por dois radicais livres triatômicos: NCN e CNN. São mostrados resultados de seções de choque diferencial e integral de polarização de spin (spin-flip) calculados na faixa de energia entre 1 a $10 \mathrm{eV}$. Para ambos os alvos, nosso estudo mostrou que a troca entre o projétil e o elétron desemparelhado do alvo é fortemente influenciada pela ocorrência de ressonâncias de forma. Como consequência disso, frações de polarização de grandes magnitudes só foram observadas na região de ressonância.
\end{abstract}

In this work, we present a theoretical investigation on spin-exchange effects in elastic electron collisions by two linear triatomic free radicals namely, NCN and CNN. Spin-polarization differential and integral cross sections calculated in the (1-10) eV energy range are reported. For both targets, our study has shown that the exchange between the scattering and the unpaired target electrons is strongly influenced by the occurrence of shape resonances. As a consequence, significant spinpolarization fractions are only observed in the resonance region.

Keywords: spin-flip, NCN, CNN, elastic collision, ISVM

\section{Introduction}

In general, low-energy electron collisions with atoms, molecules, radicals, and surfaces are strongly influenced by electron-exchange effects. Such effects can be easily characterized in the electron-impact spin-forbidden excitations (for instance, singlet-to-triplet transitions). Although exchange mechanisms are also important in lowenergy elastic electron molecule collisions, their effects are usually masked since most experimental studies are performed using unpolarized electron sources and without spin analysis of the scattered beam. It is expected that experimental studies using spin polarized electrons and with spin analysis of the scattered electrons would provide much richer information about the role of exchange in collisions, although they are more difficult to be carried out.

Despite that, few experimental studies were reported in the literature over the past years. For instance, spin-

*e-mail: milton@ fisica.ufpr.br flip (SF) differential cross sections (DCSs) for elastic electron scattering by the $\mathrm{Na}$ and $\mathrm{Hg}$ atoms as well as by the open-shell $\mathrm{O}_{2}$ and $\mathrm{NO}$ molecules were reported by Hegemann et al. ${ }^{1}$ in the (4-15) eV energy range. Significant spin-exchange effects were found for atomic targets, in accordance with the theoretical predictions. ${ }^{2,3}$ In contrast, such effects were found to be very small for elastic electron collisions with $\mathrm{O}_{2}$ and NO. These observations were in agreement with the results of Ratliff et al. ${ }^{4}$ in their measurement of average SF cross sections for scattering of electrons at thermal energies from $\mathrm{O}_{2}$ and NO molecules.

Da Paixão et al. ${ }^{5,6}$ reported theoretical DCSs for elastic collisions of polarized and unpolarized electrons with $\mathrm{O}_{2}$ and NO calculated using the Schwinger multichannel method. Their calculation provided an explanation for the small spin-exchange effects in elastic scattering from these targets as observed in the experiments. As a matter of fact, they found that although the spin-polarization fractions $\left(\mathrm{P}^{\prime} / \mathrm{P}\right)$, calculated for a given orientation of target 
in space, can deviate significantly from unity at some scattering angles, they are washed out when the molecular orientation average is performed since the calculated $\mathrm{P}^{\prime} / \mathrm{P}$ exhibit quite different angular behavior for target with different orientations. Some other calculations ${ }^{7,8}$ for $\mathrm{O}_{2}$ using different theoretical methods have also confirmed the observation by da Paixão et al.. ${ }^{5}$

Recently, we reported a theoretical investigation on spin-exchange effects in the elastic electron collisions with the open-shell $\mathrm{C}_{2} \mathrm{O}$ radical $^{9}$ using the iterative Schwinger variational method (ISVM) ${ }^{10,11}$ In that study, we have shown that the exchange effects are strongly enhanced by the occurrence of shape resonances. In this sense, the calculated P'/P averaged over all orientations are no longer isotropic and deviate significantly from unity particularly at large scattering angles. More recently, Tashiro ${ }^{12}$ investigated the exchange effects in elastic collisions of spin-polarized electrons to several open-shell diatomic molecules with ${ }^{3} \Sigma_{g}^{-}$symmetry using the R-matrix method. His study has confirmed our observation.

Physically, the occurrence of shape resonances can be understood as a temporary trapping of the scattering electron by the potential barrier of the targets. This trapping would significantly enhance both the direct and exchange interactions between the projectile and the target. Consequently, the amplitudes of resonant scattering would be significantly different from those of non-resonant scattering. For collisions between electrons and open-shell targets, more than one spin-coupling scattering channel can take place. Therefore, in the incident energy region where the shape resonance is present for only one of the spin-specific scattering channels, the difference between the scattering amplitudes of the scattering channels with different spin-couplings would increase leading to a pronounced spin-flip effect. However, if the resonances occur for both spin-specific scattering channels in the same energy region, the enhancement of the projectiletarget interaction, due to the trapping, would affect the scattering amplitudes of both channels and therefore a sort of compensation may take place and spin-flip effects could be masked. Particularly for $\mathrm{C}_{2} \mathrm{O}$ radical, the resonance positions in the ${ }^{4} \Pi$ and ${ }^{2} \Pi$ scattering channels are shifted from each other, resulting in the pronounced SF DCSs.

Here, we extend the spin-exchange study to two linear triatomic open-shell molecules, namely $\mathrm{CNN}$ and $\mathrm{NCN}$. These two targets are isoelectronic to $\mathrm{C}_{2} \mathrm{O}$ radical with the ground-state symmetry of ${ }^{3} \Sigma^{-}$. As in $\mathrm{C}_{2} \mathrm{O},{ }^{13}$ strong shape resonances are also present in the doublet- and quartet-coupling scattering channels for both targets in the low-incident energy range. ${ }^{14}$ For NCN, two sharp resonant features located at 3.3 and $4.7 \mathrm{eV}$, respectively, were observed in the spin-averaged integral cross sections (ICSs), whereas in the ICSs of elastic electron-CNN scattering, only a broad resonance located at about $2.2 \mathrm{eV}$ with a shoulder at about $3.5 \mathrm{eV}$ incident energy is seen. Probably, the presence of such resonances may also influence the spin-exchange processes during the collision. It would be interesting to investigate the behavior of the spin-polarization fractions whether the resonances are shifted or overlapped.

In this work, we report calculations of SF DCSs and spin-polarization fractions (SPFs) for elastic electron scattering by CNN and NCN in the (1-10) eV energy range. The comparison of our calculated SF DCSs and SPFs as well as the SF ICSs for these two targets should provide insight into the role played by the spin-exchange mechanisms in the collision dynamics.

\section{Theory and calculation}

Within the fixed-nuclei framework, the DCSs for electron-molecule scattering, averaged over the molecular orientations, are given as:

$$
\frac{d \sigma}{d \Omega}=\frac{1}{4 \pi} \int_{0}^{4 \pi} d \hat{R}\left|f^{L F}\right|^{2}
$$

where $f^{L F}$ is the scattering amplitude in laboratory frame $(L F), \hat{R}$ represents the orientations of the principal axis of the molecule in $L F$. Since the spherical harmonics form a complete set, equation 1 can be rewritten as:

$\frac{d \sigma}{d \Omega}=\sum_{j, m_{j}}\left\langle 00\left|f^{L F^{*}}\right| j m_{j}\right\rangle\left\langle j m_{j}\left|f^{L F}\right| 00\right\rangle$

where

$$
\left\langle\hat{R} \mid j m_{j}\right\rangle=Y_{j, m_{j}}(\hat{R})
$$

are the usual spherical harmonic functions, and

$$
Y_{0,0}(\hat{R})=(4 \pi)^{-\frac{1}{2}}
$$

The term $\left\langle j m_{j}\left|f^{L F}\right| 00\right\rangle$ appearing in equation 2 is the scattering amplitude for the rotational excitation from the fundamental state $|00\rangle(\mathrm{j}=0$ and $\mathrm{mj}=0)$ to an excited state $\left|j m_{j}\right\rangle$ in the adiabatic nuclei-approximation. In an actual study, it is convenient to calculate the body-frame (BF) transition $T$ matrix where the principal axis of the target is chosen as the $\mathrm{z}$-axis of the coordinate system. The electronic part of amplitudes $f^{L F}$ appearing in equation 2 can be related to the corresponding BF $T$ matrix by usual frame transformation. ${ }^{15}$

In this work, the electron-molecule interaction dynamics is represented by a static-exchange-polarization potential 
$V^{S E P}$, composed by the static $\left(V_{s t}\right)$, the exchange $\left(V_{e x}\right)$ and the correlation-polarization $\left(V_{c p}\right)$ components. Here, the manybody interactions are represented by an effective interaction potential $V_{c p}$. Therefore, the resulting one-electron Lippmann-Schwinger scattering equation is solved using the iterative Schwinger variational method..$^{10}$ Using this approximation, the occurrence of one-electron resonance (shape resonance) which describes a temporary trapping of the scattering electron by a potential barrier, formed by a combination of the attractive $V^{S E P}$ and a repulsive centrifugal barrier, is well represented. Nevertheless, our formalism is unable to describe Feshbach resonances, which are of many body nature. The contributions of $V_{s t}$ and $V_{e x}$ are derived exactly from a restricted open-shell Hartree-Fock self-consistent-field (SCF) target wave function. ${ }^{10,16} \mathrm{~A}$ parameter-free model potential introduced by Padial and Norcross ${ }^{17}$ is used to account for correlationpolarization contributions. In this model, a short-range correlation potential between the scattering and target electrons is defined in an inner interaction region and a long-range polarization potential in an outer region. The first crossing of the correlation and polarization potential curves defines the inner and outer regions. The short-range correlation potential is derived using the target electronic density according to equation 9 of Padial and Norcross. ${ }^{17}$ In addition, an asymptotic form of the polarization potential is used for the long-range electron-target interactions. Dipole polarizabilities are needed to generate the asymptotic form of $V_{c p}$. Since there are no experimental and/or theoretical values of dipole polarizabilities available in the literature for $\mathrm{CNN}$ and $\mathrm{NCN}$, they were calculated in this work at the single- and double-excitation configuration-interaction level of approximation. No cut-off or other adjusted parameters are needed in the calculation of $V_{c p}$.

Since CNN and NCN are open-shell targets with $X^{3} \Sigma$ ground-state electronic configuration, the coupling of the incident electron with the two unpaired $2 \pi$ electrons of the target leads to two spin-specific scattering channels, namely, the doublet $(S=1 / 2)$ and quartet $(S=3 / 2)$ couplings. The main difference between the doublet and quartet scattering channels is reflected on the treatment of the electron-exchange term in the potential operator. The interaction potential used in our calculations is expressed as

$$
V^{S}=V_{n}+V_{c p}+\sum_{\phi}\left(2 J_{\phi}-K_{\phi}\right)+J_{2 \pi^{+}}+J_{2 \pi^{-}}+a K_{2 \pi^{+}}+a K_{2 \pi^{-}}
$$

where $V_{n}$ represents the attractive potential between the projectile electron and the nuclei; $J$ and $K$ are the usual Coulomb and exchange operators $;{ }^{10}$ the index $\phi$ enumerates the doubly occupied orbitals of the target, and the parameter $a$ is 0.5 for doublet coupling and -1.0 for quartet coupling.
Further, the spin-specific Lippmann-Schwinger equation is solved using the iterative Schwinger variational method. ${ }^{10}$ The continuum wave functions are single-center expanded as:

$$
\chi_{\vec{k}}^{ \pm}(\vec{r})=\left(\frac{2}{\pi}\right)^{\frac{1}{2}} \sum_{l m} \frac{i^{l}}{k} \chi_{k l m}^{ \pm}(\vec{r}) Y_{l m}(\hat{k})
$$

where the superscripts $(-)$ and $(+)$ denote the incomingand outgoing-wave boundary conditions, respectively, $\hat{k}$ is the angular part of the linear momentum of the scattering electron.

For NCN, the truncation of the partial-wave expansion of $T$-matrix elements is limited at $l_{\max }=70$ and $m_{\max }=16$. The calculation of the spin-specific DCSs follows the scheme defined in equations 1 and 2 . On the other hand, since CNN is a polar target, the long-range dipole interaction between the target and projectile electron would lead to slow falloff of large partial-wave $T$-matrix elements. Thus, we have limited the partial-wave expansion of $T$-matrix elements up to $l_{\text {max }}=50$ and $m_{\max }=16$ for CNN, specifically for $|00\rangle(\mathrm{j}=0$ and $\mathrm{mj}=0)$ to $\left|1 m_{j}\right\rangle$ transitions, a Born-closure formula was used to account for the contribution of higher partial-wave components to the scattering amplitudes. Accordingly, the $T$ matrix is rewritten as

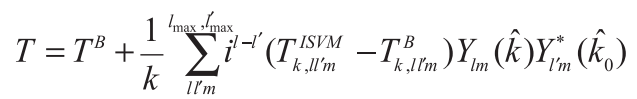

where $l=l^{\prime} \pm 1, T^{B}$ is the complete point-dipole first-Bornapproximation (FBA) $T$-matrix, $T_{k, l^{\prime} m}^{I S M}$ are the partial-wave $T$-matrix elements calculated via ISVM and $T_{k, l^{\prime} m}^{B}$ are the corresponding partial-wave point-dipole FBA $T$-matrix elements, given by

$T_{k, l^{\prime} m}^{B}=-\frac{D}{L}\left[\frac{(L+m)(L-m)}{(2 L+1)(2 L-1)}\right]^{\frac{1}{2}}$

where $D$ is the target electric dipole moment and $L=l^{\prime}$ when $l^{\prime}=l+1$, and $L=l$ when $l^{\prime}=l-1$.

Therefore, the spin-specific DCSs for electron-CNN scattering are given as a sum of the rotational excitation DCSs as:

$$
\left(\frac{d \sigma}{d \Omega}\right)^{S}=\sum_{j=0}\left(\frac{d \sigma}{d \Omega}\right)^{S}(j \leftarrow 0)
$$

where the spin-specific rotational excitation DCSs.

In order to avoid the divergence of the spin-specific rotational excitation DCSs at the forward direction, proper rotational excitation energies are taken into account in the calculation of these DCSs using the dipole-Born approximation as:

$$
\left(\frac{d \sigma}{d \Omega}\right)^{S}(j \leftarrow 0)=\frac{k_{j}}{k_{0}} \sum_{m_{j}}\left|f_{j m_{j} \leftarrow 00}^{S}\right|^{2}
$$


where $k_{j}$ and $k_{0}$ are the final and initial linear momenta of the scattering electron, respectively. In equation $10, f^{S}$ is the spin-specific fixed-nuclei electron scattering amplitude in the laboratory-frame (LF) and $S$ is the total spin of the (electron + target) system.

The spin-averaged DCSs for elastic electron scattering by $\mathrm{CNN}$ and $\mathrm{NCN}$ are calculated using the statistical weight for doublet (2/6) and quartet (4/6) scattering channels, as

$$
\left(\frac{d \sigma}{d \Omega}\right)=\frac{1}{6}\left[4\left(\frac{d \sigma}{d \Omega}\right)^{\frac{3}{2}}+2\left(\frac{d \sigma}{d \Omega}\right)^{\frac{1}{2}}\right]
$$

Moreover, the SF DCSs can be defined in terms of the spin-specific electron scattering amplitudes by

$$
\left(\frac{d \sigma}{d \Omega}\right)_{S F}=\frac{4}{27}\left|f^{\frac{3}{2}}-f^{\frac{1}{2}}\right|^{2}
$$

and the SF ICS is obtained via the integral

$$
\sigma_{S F}=\int\left(\frac{d \sigma}{d \Omega}\right)_{S F} d \Omega
$$

Finally, the SPFs are given by

$$
\frac{P^{\prime}}{P}=1-\frac{2(d \sigma / d \Omega)_{S F}}{(d \sigma / d \Omega)}
$$

In the present study, a standard triple-zeta-valence basis $\operatorname{set}^{18}$ augmented by two $\mathrm{s}(\alpha=0.04531,0.01571)$, one $\mathrm{p}$ $(\alpha=0.03237)$, and two $d(\alpha=1.573,0.2371)$ uncontracted functions for carbon atom and two s $(\alpha=0.07571,0.0293)$, two $\mathrm{p}(\alpha=0.04413,0.01137)$, and two $\mathrm{d}(\alpha=1.639,0.2193)$ for nitrogen atom is used for the calculation of the SCF wave function of the target. The results of some calculated properties are summarized in Table 1, where the SCF results of Armstrong et al. ${ }^{19}$ are also shown for comparison.

\section{Results and Discussion}

In Figures 1(a) and 1(b), we show the ICSs calculated using both the doublet and quartet coupling schemes in the (1-10) eV energy range for elastic electron scattering from
$\mathrm{NCN}$ and $\mathrm{CNN}$, respectively. For elastic $e^{-}-\mathrm{NCN}$ collisions, the sharp resonance-like feature located at about $3.3 \mathrm{eV}$ incident energy is identified as due to the occurrence of a ${ }^{4} \Pi_{u}$ shape resonance whereas that located at about $4.7 \mathrm{eV}$ is due to ${ }^{2} \Pi_{u}$ shape resonance. Similarly, the physical origin of the broad resonance centered at around $2.2 \mathrm{eV}$, in the ICS for the $e^{-}$-CNN is identified as due to the ${ }^{4} \Pi$ scattering channel and that located at $3.5 \mathrm{eV}$ as due to the ${ }^{2} \Pi$ symmetry. The shift in the resonance positions due to different spin couplings is somehow expected since the resonances are not originated from the same state. It is because in our framework the doublet and quartet collisions were treated separately. In each case, the one-particle wave function of the scattering electron distorted by the static-exchangepolarization field of the target is computed. The resonances found here can also be interpreted as a temporary occupation of a virtual orbital of the target by the scattering electron. ${ }^{20}$ Nevertheless, in our calculations the wave functions of the scattering electron in the continuum are not approximated by electronic configurations with $(\mathrm{N}+1)$ particles, but obtained directly via numerical solution of the Lippmann-Schwinger scattering equation. Thus, it is not straightforward to point out which virtual orbital is responsible for the occurrence of shape resonances.

In Figures 2 and 3 we present the calculated SF DCSs in the (1-10) eV energy range for elastic electron collisions from $\mathrm{NCN}$ and $\mathrm{CNN}$ radicals, respectively. For both targets, it is clearly seen that the SF DCSs in the resonance regions, 3-5 eV for $\mathrm{NCN}$, and 2-4 eV for $\mathrm{CNN}$, are significantly larger than elsewhere. Since the resonances in the ${ }^{4} \Pi_{u}$ and ${ }^{2} \Pi_{u}$ scattering channels in NCN are well split, the large SF DCSs in that energy range are in fact expected. On the other hand, it seems that the significant overlap of the broad resonances in $\mathrm{CNN}$ at about $3 \mathrm{eV}$ (shown in Figure 1) does not mask the important SF effects in that energy region. In order to better understand the role played by the shape resonances in SF DCSs for CNN, in Figure 4 we present two relevant partial-wave $T$-matrix elements $\left(T_{I l^{\prime} m}\right)$, namely the non-resonant $T_{110}$ and resonant $T_{221}$ components for this

Table 1. Calculated properties of CNN and NCN

\begin{tabular}{lcccc}
\hline & \multicolumn{2}{c}{ This work } & \multicolumn{2}{c}{ Armstrong et al. ${ }^{19}$} \\
& $\mathrm{CNN}$ & $\mathrm{NCN}$ & $\mathrm{CNN}$ & $\mathrm{NCN}$ \\
\hline Energy (hartree) & -147.51206 & -147.62005 & -147.494784 & -147.541177 \\
$\mathrm{R}_{\mathrm{CN}}$ (bohr) & 2.12306 & 2.31388 & 2.12310 & 2.31380 \\
$\mathrm{R}_{\mathrm{NN}}$ (bohr) & 2.26654 & $\ldots$ & 2.26655 & $\ldots$ \\
Dipole Moment (D) & 0.90406 & $\ldots$ & $\ldots$ & $\ldots$ \\
$\alpha_{0}$ (a.u.) & 23.1202 & 26.5702 & $\ldots$ & $\ldots$ \\
$\alpha_{2}$ (a.u.) & 6.5852 & 15.3116 & $\ldots$ & $\ldots$ \\
\hline
\end{tabular}



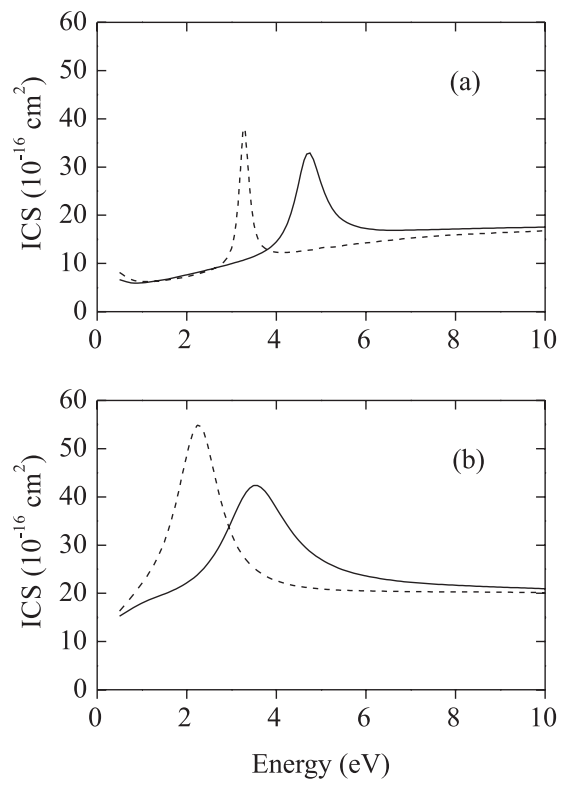

Figure 1. ICS for elastic electron scattering by (a) NCN and (b) CNN. Solid line, the results calculated for the doublet coupling; dashed line, results calculated for the quartet coupling.

target, as functions of the incident energies. It is shown that both the real and imaginary parts of the non-resonant element $T_{110}$ for the doublet-coupling scattering vary smoothly with the incident energies and are very similar to the corresponding terms for the quartet scattering. On the other hand, significant mismatches are seen between the real parts of the $T_{221}$ components for the doublet and quartet scattering, as well as between the corresponding imaginary parts in the (1-5) eV energy range. This is probably the reason for the significant SF DCS seen in Figure 3 for CNN.

In Figures 5(a) and 5(b) we show the SF ICSs in the (1-10) eV energy range calculated for $e^{-}-\mathrm{NCN}$ and $e^{-}$-CNN collisions, respectively. For NCN, two narrow resonance features similar to those seen in the elastic spin-specific ICSs shown in Figure 1(a) are found. On the other hand, for CNN, only one broad feature centered at about $2.5 \mathrm{eV}$ is seen. Out of the resonance region, the SF ICSs for both targets are very small. Therefore, the present calculations clearly indicate that the spin-exchange effects between the scattered electron and the unpaired electrons of the target are substantially enhanced by the occurrence of shape resonances. Particularly for NCN, it is seen that the energy positions of the resonances in the calculated ICSs for quartet and doublet couplings are shifted. Therefore, the peak located at about $3.3 \mathrm{eV}$ in the calculated SF ICSs is due to the occurrence of the ${ }^{4} \Pi_{u}$ shape resonance whereas that located at about $4.7 \mathrm{eV}$ is associated to the resonance in the ${ }^{2} \Pi_{u}$ scattering channel. Moreover, the resonance features seen in the spin-specific ICSs for elastic $e^{-}$-CNN scattering, shown in Figure 1(b), are much broader. Therefore, the
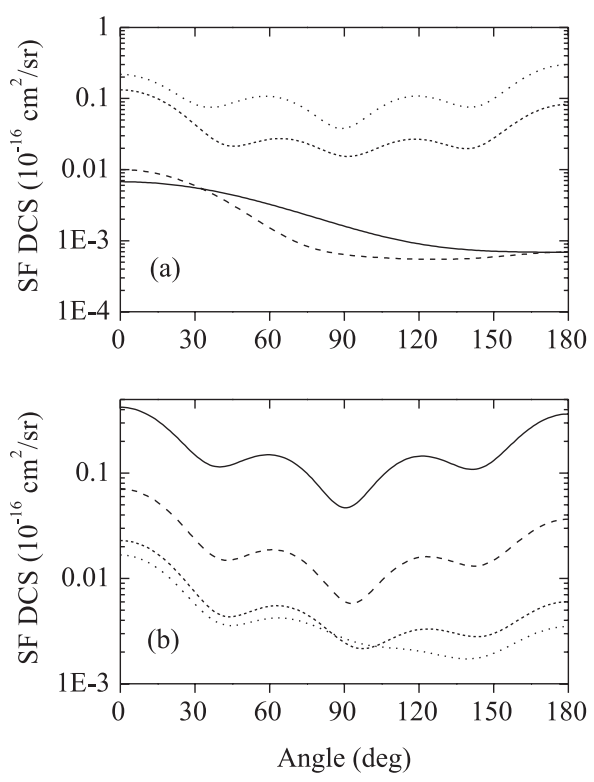

Figure 2. Calculated SF DCSs for elastic $e^{-}$-NCN collisions. (a): solid line, at $1 \mathrm{eV}$; dashed line, at $2 \mathrm{eV}$; short-dashed line, at $3 \mathrm{eV}$; and dotted line, at $4 \mathrm{eV}$. (b): solid line, at $5 \mathrm{eV}$; dashed line, at $6 \mathrm{eV}$; short-dashed line, at $8 \mathrm{eV}$; and dotted line, at $10 \mathrm{eV}$.
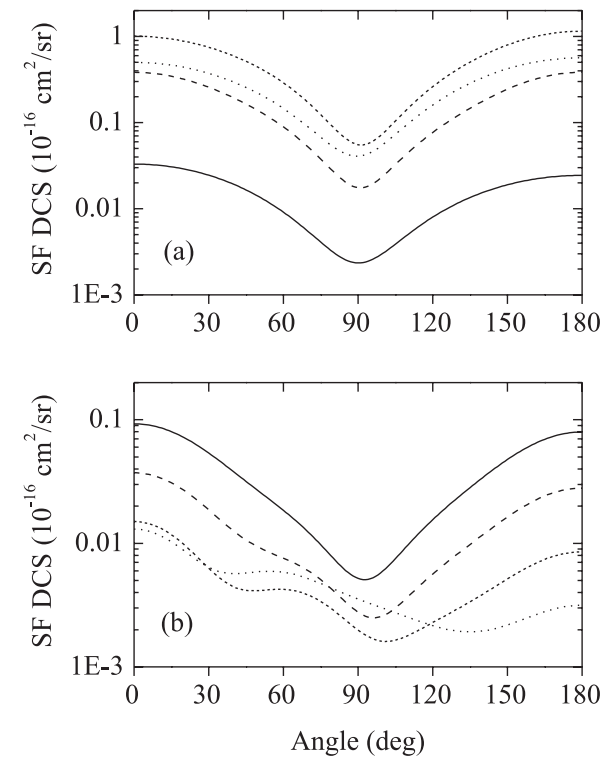

Figure 3. Calculated SF DCSs for elastic $e^{-}-\mathrm{CNN}$ collisions. (a): solid line, at $1 \mathrm{eV}$; dashed line, at $2 \mathrm{eV}$; short-dashed line, at $3 \mathrm{eV}$; and dotted line, at $4 \mathrm{eV}$. (b): solid line, at $5 \mathrm{eV}$; dashed line, at $6 \mathrm{eV}$; short-dashed line, at $8 \mathrm{eV}$; and dotted line, at $10 \mathrm{eV}$.

unique resonance feature seen in the SF ICSs for this target is due to the overlap of the spin-exchange effects associated to the resonances in both ${ }^{4} \Pi$ and ${ }^{2} \Pi$ scattering channels.

In Figures 6 and 7, we present the angular distribution of the SPFs in the (1-10) eV energy range calculated for $e^{-}$-NCN and $e^{-}-\mathrm{CNN}$ collisions, respectively. The present polarization fractions are calculated according to equation 

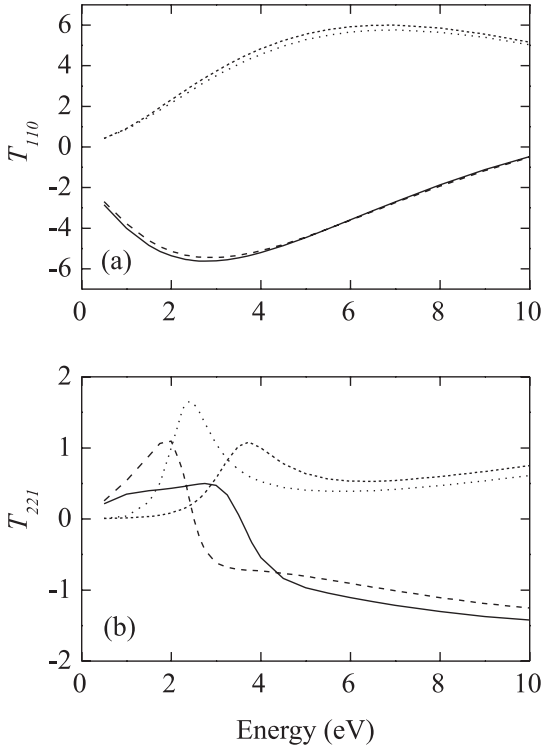

Figure 4. (a) $T_{110}$ and (b) $T_{221}$ elements for doublet and quartet elastic $e^{-}$-CNN scattering. Solid line, real part for doublet scattering; dashed line, real part for quartet scattering; short-dashed line, imaginary part for doublet scattering; dotted-line, imaginary part for quartet scattering.
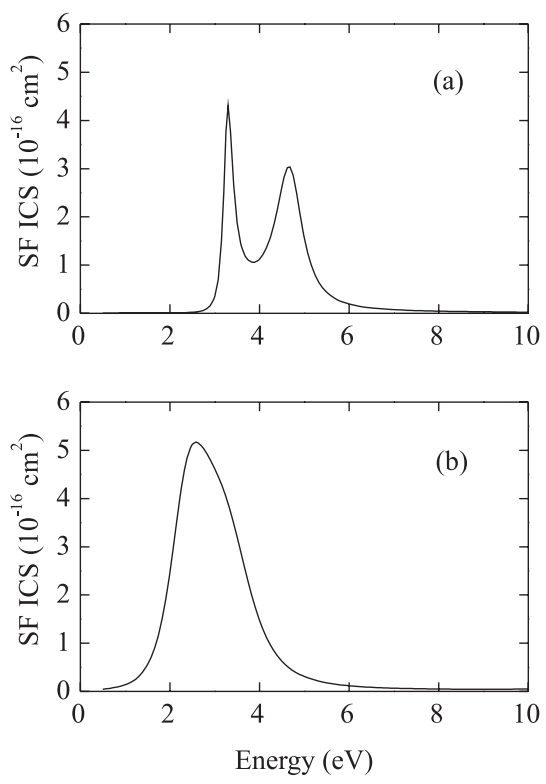

Figure 5. Calculated SF ICSs for elastic electron collisions with (a) NCN, and (b) CNN in the (1-10) eV range.

14 using the rotationally summed spin-averaged DCSs and SF DCSs. Actually, the summation over rotational transitions is equivalent to the molecular orientations averaging procedure used by da Paixão et al..$^{5}$ It is interesting to see in Figures 6 and 7 that our calculated rotationally-summed SPFs show strong oscillations in the resonance region and particularly at large scattering angles, and their magnitudes deviate significantly from 1 . These results reinforce what was observed in our previous study for elastic $e^{-}-\mathrm{C}_{2} \mathrm{O}$ collisions, ${ }^{9}$ that is, the spin-exchange effects between the scattered electron and the unpaired electrons of the target can be significantly enhanced by the occurrence of resonances.

In order to confirm the important role played by the shape resonance in the spin-exchange mechanism, Tashiro ${ }^{12}$ has carried out calculations in which the contributions of the electronic configurations responsible for the resonance were removed. The resulting SPFs are then close to unity at all incident energies covered in his work. Based on a similar idea, we also performed calculations of the SPFs, but removing the contributions of the resonant scattering
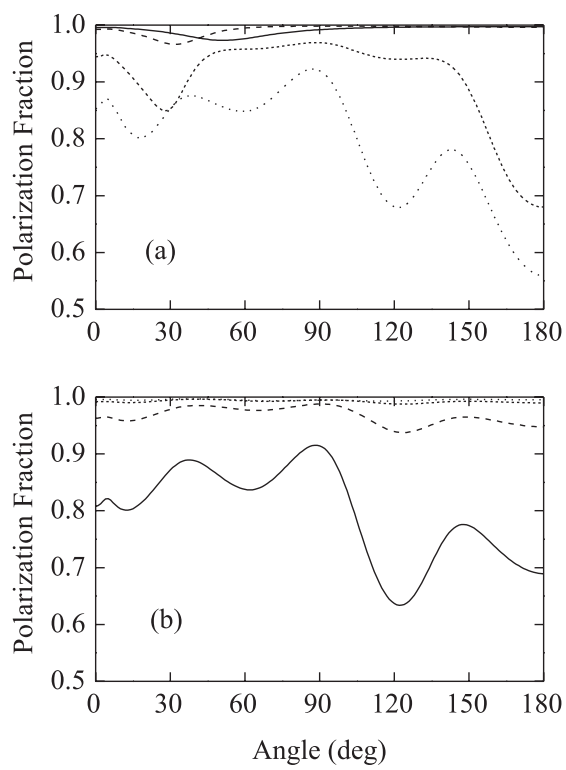

Figure 6. Angular distribution of the SPFs for elastic $e^{-}-\mathrm{NCN}$ collisions. The symbols used are the same as those in Figure 2.
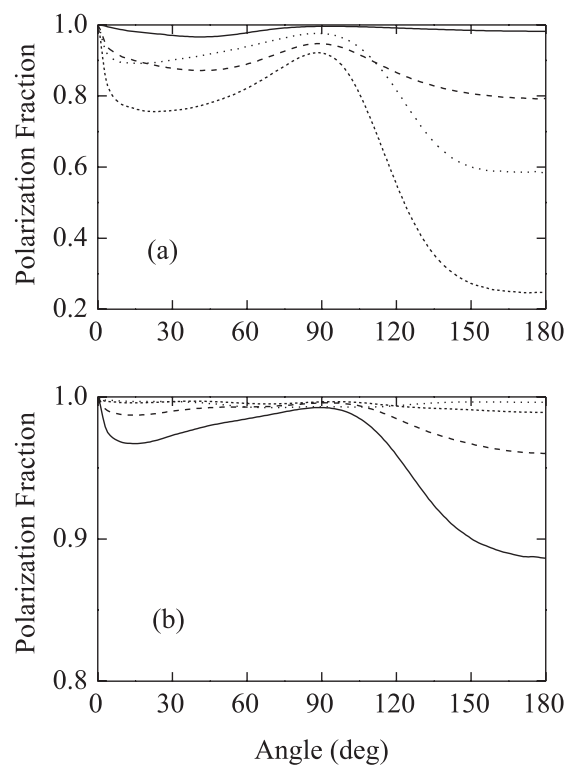

Figure 7. Angular distribution of the SPFs for elastic $e^{-}$-CNN collisions. The symbols used are the same as those in Figure 2. 
channel. Namely, the partial-wave $T$-matrix elements belonging to the resonant scattering channel were removed from the summation in equation 7. The calculation of the spin-averaged DCSs and SF DCSs are then performed following the scheme described in equations 9-12. In fact, this procedure is different from that used by Tashiro ${ }^{12}$ because in our study the contributions from both resonant and non-resonant $T$-matrix elements belonging to the same scattering channel were removed. The calculated SPFs resulting from our procedure are shown in Figure 8 for some selected incident energies. It is seen that all SPFs are nearly 1 in the entire angular range. This observation clearly indicates that the depolarization of the scattered electrons is in fact directly associated with the resonances.
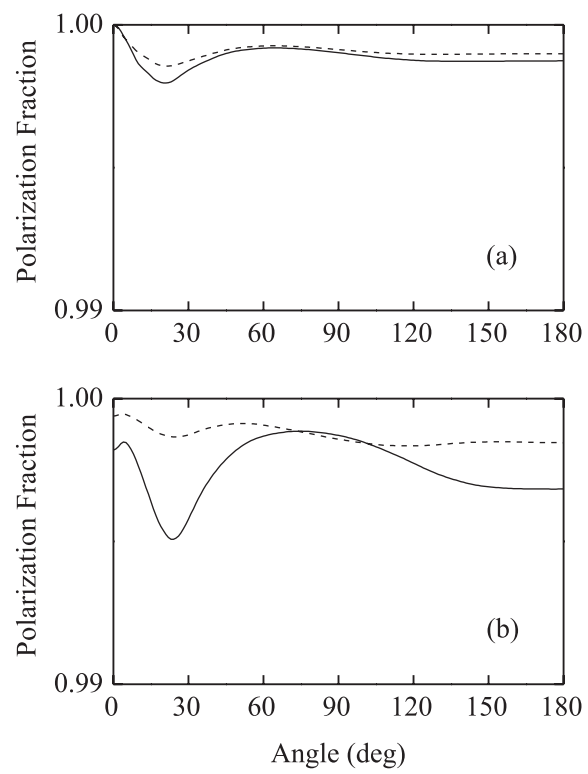

Figure 8. Angular distribution of the SPFs with the resonant scattering channel removed (a) for elastic $e^{-}-\mathrm{CNN}$ collisions. Solid line, at $2 \mathrm{eV}$; dashed line, at $3 \mathrm{eV}$; and (b) for elastic $e^{-}-\mathrm{NCN}$ collisions. Solid line, at $3 \mathrm{eV}$; dashed line, at $5 \mathrm{eV}$.

In our previous study for $\mathrm{C}_{2} \mathrm{O}$, we stated that the shift of the resonance positions associated with distinct spin couplings has lead to the large SF DCSs as well as the significant deviation of the rotationally-summed SPFs from unity in the resonance region. However, it is found in the present study that the influences of shape resonances on the spin-exchange processes are even more extensive, since significant SF effects also took place despite the overlap of resonance positions associated with the doublet and quartet couplings in CNN. On the other hand, our method is unable to predict the occurrence of Feshbach resonances. However, it is expected that the presence of such resonances will not affect the important role of the shape resonances in spin-flip processes. Therefore, the conclusion would be the same.

\section{Conclusions}

In summary, the present work reports a theoretical investigation on the spin-exchange processes in the low-energy elastic electron collisions on $\mathrm{CNN}$ and $\mathrm{NCN}$ radicals. Our calculation has confirmed that the exchange between the scattering and unpaired target electrons can be strongly enhanced by the occurrence of shape resonances. In this sense, calculated rotationally-summed SPFs, which is equivalent to the molecular orientation averaged results, ${ }^{5}$ deviate significantly from unity in the resonance region. Probably, the small spin-exchange effects observed in the previous theoretical and experimental elastic electron scattering by $\mathrm{NO}$ and $\mathrm{O}_{2}$ are due to the lack of strong resonances at the incident energies where the studies were carried out. Although the shift of resonance positions associated with different spin couplings can certainly contribute to the significant SF DCSs, the spin-exchange effects are also sensitive to the relative phases between the $f^{3 / 2}$ and $f^{1 / 2}$. Therefore, significant depolarization may happen even for systems with spin-specific resonances lying at about same incident energies, as verified for CNN, which is interesting.

\section{Acknowledgments}

This research was partially supported by the Brazilian Agencies: CNPq, FUNPAR, Fundação Araucária, FINEP/ PACDT-UFPR and FAPESP.

\section{References}

1. Hegemann, T.; Oberste-Vorth, M.; Vogts, R.; Hanne, G. F.; Phys. Rev. Lett. 1991, 66, 2968.

2. Moores, D. L.; Comput. Phys. Commun. 1971, 2, 360.

3. Bartschat, K.; Madison, D. H.; J. Phys. B: At., Mol. Opt. Phys. 1987, 20, 1609.

4. Ratliff, J. M.; Rutherford, G. H.; Dunning, F. B.; Walters, G. K.; Phys. Rev. A 1989, 39, 5584.

5. da Paixão, F. J.; Lima, M. A. P.; McKoy, V.; Phys. Rev. Lett. 1992, 68, 1698.

6. da Paixão, F. J.; Lima, M. A. P.; McKoy, V.; Phys. Rev. A 1996, 53,1400 .

7. Woste, G.; Higgins, K.; Duddy, P.; Fullerton, C. M.; Thompson, D. G.; J. Phys. B: At., Mol. Opt. Phys. 1996, 29, 2553.

8. Machado, L. E.; Ribeiro, E. M. S.; Lee, M.-T.; Fujimoto, M. M.; Brescansin, L. M.; Phys. Rev. A 1999, 60, 1199.

9. Fujimoto, M. M.; Michelin, S. E.; Iga, I.; Lee, M.-T.; Phys. Rev. A 2006, 73, 012714 .

10. Lucchese, R. R.; Raseev, G.; McKoy, V.; Phys. Rev. A 1982, 25 , 2572. 
11. Lee, M.-T.; Iga, I.; Homem, M. G. P.; Machado, L. E.; Brescansin, L. M.; Phys. Rev. A 2002, 65, 062702.

12. Tashiro, M.; Phys. Rev. A 2008, 77, 012723.

13. Fujimoto, M. M.; Lee, M.-T.; Michelin, S. E.; Phys. Rev. A 2004, 69, 052706.

14. Michelin, S. E.; Oliveira, H. L.; Kroin, T.; Soares, L. S. S.; Veiteinheimer, E.; Luz, C. A. R.; Lima, M. F.; Fujimoto, M. M.; Lee, M.-T.; J. Chem. Phys. 2005, 122, 094309.

15. Rose, M. E.; Elementary Theory of Angular Momentum, John Wiley: N.Y., 1957.
16. Faisal, F. H. M.; J. Phys. B: At., Mol. Opt. Phys. 1970, 3, 636.

17. Padial, N. T.; Norcross, D. W.; Phys. Rev. A 1984, 29, 1742.

18. Dunning, T. H.; J. Chem. Phys. 1971, 55, 716.

19. Armstrong, J.; Degoricija, L.; Hildebrand, A.; Koehne, J.; Fleming, P. E.; Chem. Phys. Lett. 2000, 332, 591.

20. Piancastelli, M. N.; J. Electron Spectrosc. Relat. Phenom. 1999, 100, 167.

Received: January 22, 2009

Web Release Date: November 12, 2009

FAPESP helped in meeting the publication costs of this article. 